Review Article

\title{
Biological variations, technical stability and elution tactics in dried blood spots specimens: neonatal screening outcomes
}

\author{
Subhasree. C. R. ${ }^{1}$, Subramaniam S. ${ }^{2}$, Shyama S. ${ }^{3}$ \\ ${ }^{1}$ Subhasree C.R., Research Scholar, ${ }^{2}$ Samu Subramaniam, Lab Director and Head of the Department; above authors are \\ attached with Department of Biochemistry, Regenix Super Speciality Laboratories Private Ltd, Chennai, India, ${ }^{3}$ Shyama \\ Subramaniam, Professor, Consultant, Lab services, Apollo Hospitals, Chennai, Tamil Nadu, India.
}

Corresponding Author: Samu Subramaniam, Lab Director and Head of the Department, Department of Biochemistry, Regenix Super Specialty Laboratories Private Ltd, Chennai, India, E-mail: Subramaniam@regenix.in

\begin{abstract}
For many years, pre-analytical variables were only inappropriately considered in the field of dried blood spots (DBS) testing and even today, with the exception of newborn screening, the entire pre-analytical phase, which comprises the preparation and processing of DBS for their final analysis has not been standardized. (1) compared to conventional venepuncture, less blood volume is required and this fact was most important in paediatric diagnostics; (2) blood collection is simple, non-invasive, and inexpensive; (3) the risk of bacterial contamination or haemolysis is minimal; and (4) DBS can be preserved for long periods with almost no deterioration of the analytes. Advances techniques applied to diagnose the disorders of Inborn errors of metabolism. Dried blood spots (DBSs) have potential use in remote health applications for individual and population diagnosis, and can enable epidemiological surveillance for known and unknown diseases. DBS sampling offers great potential for remote health applications as a cost-effective sample format for large-scale screening and diagnostic purposes, but the DBS samples often need to be transported from remote sampling locations (e.g., tropical or developing countries) to analytical laboratories where the quantitative measurements of different metabolic or disease biomarkers can be carried out. Dried blood spots are commonly method for collecting, storing, transporting, and analysing a variety of human body fluids. Since 2006, however a variety of new and innovative dried blood spots applications, although this pre-analytical approach represents an interesting alternative to classical venous blood sampling, its routine use is limited. Here, we review the application of DBS technology in clinical chemistry, molecular assays, small molecules, and large molecules all are perfectly suited to evaluate its future role supported by new analytical methods such as mass spectrometry.
\end{abstract}

Keywords: Inborn Errors of Metabolism; Diagnosis; Genetic; Newborn screening; Paediatric; Venepuncture; Dried Blood Spots

\section{Introduction}

Robert Guthrie, introduced the first new-born screening card in the United States for Phenylketonuria in the early 60's and the use of the Guthrie card to collect from the heel prick blood using specified lancet for neonatal Screening is now standard. He begins his clinical career as cancer Research doctor but became interested in preventing mental retardation and developmental disabilities for his second son and his niece diagnosed with Phenylketonuria (PKU). Dried blood spots is commonly method for collecting, storing, transporting, and analysing a variety of human body

Manuscript received: $30^{\text {th }}$ June 2019

Reviewed: $10^{\text {th }}$ July 2019

Author Corrected: $16^{\text {th }}$ July 2019

Accepted for Publication: $23^{\text {rd }}$ July 2019 fluids. Newborn screening is a simple blood test to identify neonates who may have one of 29 primary treatable rare inherited disorders. A newborn baby can look healthy but one of these rare disorders. The most common disorders are congenital hypothyroidism $(\mathrm{CH})$, cystic fibrosis (CFTR), medium chain acyl coA deficiency (MCAD), maple syrup urine disease (MSUD), congenital adrenal hyperplasia (CAH), biotinidase deficiency (BIOT), phenylketonuria (PKU), galactosemia (GALT) and glucose-6-phosphatase deficiency (G-6-PD). Newborn screening aims at the earliest possible recognition of disorders to prevent the most serious consequences by timely intervention screening is not a confirmatory diagnosis and requires 
Review Article

further investigations [1]. These diseases will be included in the first case, because the annual birth rate is 21.76 births /1000 population and in Delhi alone, nearly 900 births takes place every day; considering this figure there may be one or two babies born Delhi alone with a metabolic defect each day (Info change and India News 2010). One in every 1,000 newborns has inborn errors of metabolism (IEM) or other disorders that can

Table-1: Burden of genetic diseases in India: recent studies (2015).

\begin{tabular}{|l|c|c|}
\hline Disorder & Incidence & Births/year \\
\hline Congenital malformation & $1: 50$ & 678,000 \\
\hline Down's syndrome & $1: 800$ & 34,000 \\
\hline Metabolic disorders & $1: 1200$ & 22,500 \\
\hline Beta-thalassemia and sickle cell disease & $1: 1700$ & 16,700 \\
\hline Congenital hypothyroidism & $1: 2500$ & 10,900 \\
\hline Duchenne muscular dystrophy & $1: 10000$ & 2,700 \\
\hline Spinal muscular atrophy & $1: 10000$ & 2,700 \\
\hline
\end{tabular}

Dried blood sampling has been used to screen neonates for endocrine disorders and metabolic disorders for over 50 years. The blood spots collected on specialized filter paper "what man 903 grade protein saver card" for neonatal screening. Each circle holds 70-80 microliter $(\mu \mathrm{l})$ of whole blood sample. A spot of blood were collected from a heel stick, dried it for one hour in the air, ease of transport and sample stability, Once dried the sample is stable for months to years at ambient temperature or under refrigeration [3,4]. A spot of blood from a heel stick of the infant is placed on filter paper and allowed to dry, eluted with specific solvent and analysed for specific disorders. A circular punch (about $3 \mathrm{~mm}$ ) is removed, eluted with solvent and analysed for metabolic markers, mainly due to LS-MS/MS [5]. Advantages of DBS sampling include minimal volume requirement (approximately 20-70 microliter) per spot, ease of sampling by heel stick lancet with speculated for new born screening 1.5-2.2 mm depth which is suitable for newborn screening. This DBS sampling methodology also used for gene sampling, a long-term genetic sampling and bio-banking DNA and RNA specimens.

Biological variations: Specimen transport and logistics are simplified as, apart from the advantage of avoiding venepuncture. DBS Specimen handling doesn't need high skill and biohazard risk is thought to be less. Minimal training required for sample collection from newborns, because of the sensitivity and to reduce the artefacts due to uneven sampling; touching the paper; to validate large or small of a drop or too much time between drops can make a sample not suitable for analysis. After specimens has arrived for the laboratory testing, a small punch (3-6 mm diameter) is taken from card, eluate in solvent (separate buffers) for each analyte and analysed by standard analytical methods.Put the filter cards on a clean paper towel in a biohazard safety cabinet and let them dry, preferably for (but for at least $1 \mathrm{hr}$ ), at room temperature. When the drying process is complete, the blood spots have a uniformly dark brownish colour. Allow the sufficient quantity of blood to soak through to completely fill the pre-printed circle on the filter paper (Figure 1).

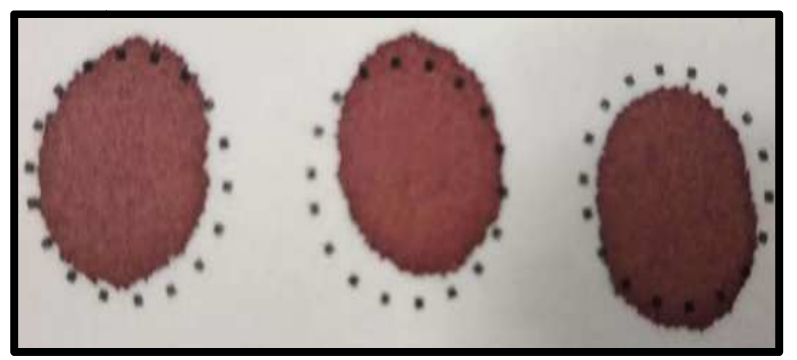

Figure-1: Valid specimen form-heel prick-dried blood specimens 
Technical stability: DBS should be transferred to -20 degree celsius or lower as soon as possible. However, when this is not possible they can be kept or transport at ambient temperature up to 14 days after collection.

After this time, DBS must be either processed for genotyping or frozen at -20 degree celsius or below. As humidity and UV light can damage DBS, always keep them in zip lock bags, with desiccant in the dark [6].

DBS cannot be kept or transported at ambient temperature for longer than 14 days. If the sampling and analysing cannot be performed within the 14 days from the date of collection, DBS Should be transported to a central facility ( $\left.-70^{\circ} \mathrm{C}\right)$ freezer. Even minimal bias when introduced to a microsample will have a large effect on the eventual measurement.

It may be the case that clinical application of DBS is better served by developing diagnostics that are especially suited to DBS rather than attempting to apply DBS measurements to diagnostics originally developed for other samples (Table2).

\section{Table-2: Biological variations andtechnical stability of dried blood samples}

\begin{tabular}{|c|c|}
\hline Biological variations & Technical Stability \\
\hline Easy and inexpensive to collect & Very small volume of material \\
\hline Initially easy to store & Pre-processing may increase lab costs \\
\hline Potentially easy to ship & $\begin{array}{l}\text { Storage problems- Prone to moldand need to be stored with } \\
\text { desiccant. }\end{array}$ \\
\hline $\begin{array}{l}\text { Small samples sizes used, usually generated } \\
\text { from a finger prick }\end{array}$ & $-70^{\circ} \mathrm{C}$ for nucleic acids studies (viral load and genotyping) \\
\hline No centrifugation required & $\begin{array}{l}\text { On occasion there is not enough sample for performing } \\
\text { certain test }\end{array}$ \\
\hline $\begin{array}{l}\text { DBS is not considered to be biohazard, so the } \\
\text { sample may be collected of site and easily } \\
\text { transported to laboratory. }\end{array}$ & $\begin{array}{l}\text { Handling is more difficult than whole blood when } \\
\text { performing the test. Uncertainty about long term stability }\end{array}$ \\
\hline Simplified process and better quality & Hematocriteffect \\
\hline $\begin{array}{l}\text { Due to moisture removal greater analyte } \\
\text { stability, especially for enzyme - sensitive } \\
\text { compounds }\end{array}$ & Sample inhomogeneity \\
\hline Federal quality assurance program & Time, space, and labour-intensive processing \\
\hline Good precision and reproducibility & Non- traditional sample matrix \\
\hline Wide range of analytes validated & Chromatographic effects \\
\hline Reduced biohazard risk & Differential analyte stability \\
\hline Personalized clinical reference ranges & Requiring drying \\
\hline Reduced material input and waste & Poorly defined regulatory landscape \\
\hline
\end{tabular}

Elution tactics for dried blood specimens for neonatal screening: Blood samples are collected on absorbent paper as dried blood spots for many medical purposes, for proteins and nucleic acids and amino acids [7], Toxicology [8] and Serology [9].

DBS facilities logistics and centrifuging. For most of the epidemiological studies, dried blood spots work station is more efficient for accuracy of the work. Here the simple procedure for elution of the sample for each analyte, different protocols has to be followed.

The rapid new technologies involved in the newborn screening, a uniform panel and system about the disorders that are required to offer nationwide. To provide the uniformity of screening panel, newborn screening laboratories testing 
Review Article

procedures do not identify the biochemical markers rather than specific analytes that are related to the disorders. The expert panel identified primary as 29 metabolic disease conditions for which neonatal screening should be mandatory.

An additional 25 conditions were identified because they are part of the differential diagnosis of a condition in the core panel, they are clinically significant and revealed with screening technology but lack an efficacious treatment, or they represent incidental findings for which there is potential clinical significance [10](Table 3).

In enzyme linked lmmunosorbentassay (ELISA) methods: for congenital hypothyroidism (neonatal thyroid stimulating hormone) eluting the hormones using with microplate coated with peroxidase labelled with anti TSH monoclonal antibody, the assay is based on the sandwich enzyme linked immunoassay.

For congenital adrenal hyperplasia (CAH/21-hydroxylase deficiency) neonatal 17OHP determination, using sandwich ELISA microplate was coated with anti-rabbit IgG antibody along with peroxidase labelled 17-OHP.

Glucose -6- phosphate dehydrogenase Enzyme (G-6PD): Buffered solution with $0.1 \%$ Sodium Azide as preservative. Galactose- 1- phosphate Uridyl transferase (GALT): Buffered Solution with 33\% Trichloroacetic acid.

For immunofluorescent assay methods [11], ammonium increases the elution without damaging the antibodies: using tween 20/80, sodium azide, hydrogen peroxide increased or decreased with elution temperatures, violent shaking and strong pestling.

For enzyme linked fluorescent assay methods, $0.2 \% \mathrm{NH}_{3}(\mathrm{pH} 10.9$ ), improves the elution, Liquid ammonium (NH3, a weak base) to determine the minimum effective concentration necessary for elution and to the effect on IgG quantity. $0.2 \%$ ammonia ( $2.5 \mu 1$ of a $25 \% \mathrm{NH}_{3}$ Solution) was sufficient for a much-improved blood elution [12].

The elution in phosphate buffered saline, $\mathrm{pH}$ level of which was raised to 10.85 using sodium hydroxide, was compared to be better elution in normal PBS (pH 7. 43) and PBS- $\mathrm{NH}_{3}(\mathrm{pH} 10.85)$.

For sickle cell disease and Beta-thalassemia, using high performance -liquid chromatography (HPLC), DBS was eluted with a haemolysing Solution, $2.5 \mathrm{~g} / \mathrm{L}$ ethylene diamine tetracetic acid (EDTA), $50 \mathrm{mg} / \mathrm{L}$ potassium cyanide (KCN) and haemoglobin variants were separated on a salt gradient obtained by mixing buffered (bris- tris $20 \mathrm{mmol} / \mathrm{L}, \mathrm{KCN} 2$ $\mathrm{mmol} / \mathrm{L}, \mathrm{NaCl} 200 \mathrm{mmol} / \mathrm{L}, \mathrm{pH}-6.55$ ) and buffers (bris -tris $20 \mathrm{mmol} / \mathrm{L}, \mathrm{KCN} 2 \mathrm{mmol} / \mathrm{L}, \mathrm{NaCl} 200 \mathrm{mmol} / \mathrm{L}, \mathrm{pH}-6.55$ ), the flow rate was $1 \mathrm{ml} / \mathrm{min}$.

For molecular analysis, dried blood samples are a micro sampling alternative to traditional plasma or serum sampling for pharmacology works.

For extraction and estimation of DNA or RNA from dried blood spots on filter paper ( $3 \mathrm{~mm}$ FTA card), extraction solution has ethanol and chatrophic agents, that can disrupt the hydrogen bonding network between water molecules.

In clinical chemistry metabolite panel, phosphate buffer saline with 1\% tween (PBST), $0.9 \%$ sodium chloride, and 5\% trichloroacetic acid (TCA) for the extraction of sample from the dried blood spots (DBSs).

In the recent year's large molecule, small molecule and elemental analysis, liquid chromatography- mass spectrometry (LC-MS/MS)[13], there has also been an increased interest in the use of DBS as a cost - effective replacement for blood plasma and serum in studies involving proteomics or metabolomics as well as pharmaceutical research and development for therapeutic drug monitoring, pharmacokinetics or toxicokinetic.

The WHO recommended FDA approved DBS collection cards as considered medical devices for blood specimen collection include What man 903 protein saver card and What man FTA DMPK -A, B, C and D cards, all of which utilize cellulose as an absorbing matrix and Perkin Elmer 226 (Ahlstrom grade 226) spot saver cards, Manufactured from cotton linter by perkinelmer for inborn errors of metabolic disorders screening. 
Table-3: Dried blood spots methodologies involved in the uniform newborn screening panel (inborn errors of metabolism).

\begin{tabular}{|c|c|}
\hline Core Condition & Analytes as markers \\
\hline \multicolumn{2}{|c|}{ Enzyme linked Immunosorbent assay /DELFIA /Fluorometry } \\
\hline \multicolumn{2}{|l|}{ Basic newborn screening panel } \\
\hline Congenital hypothyroidism & Thyroid Stimulating Hormone \\
\hline Congenital adrenal hyperplasia & $17 \alpha$ - hydroxyprogesterone \\
\hline Glucose -6 -phosphate dehydrogenase deficiency & G-6-PD enzyme \\
\hline Cystic fibrosis & Immuno-reactive Trypsinogen \\
\hline Classic galactosemia & Galactose -1-phosphate uridyltransferase (GALT) \\
\hline Biotinidasedeficiency & Biotinidase enzyme \\
\hline Homocystinuria & Methionine \\
\hline \multicolumn{2}{|c|}{ Liquid chromatography- Mass spectrometry/ Mass spectrometry } \\
\hline \multicolumn{2}{|l|}{ Expanded newborn screening panel (metabolic disorders) } \\
\hline Organic acid disorders & Small and large molecule analytes \\
\hline Propionic acidemia & Propionyl C3个 \\
\hline $\begin{array}{l}\text { Methylmalonic } \quad \text { acidemia(methylmalonyl-CoA } \\
\text { mutase) }\end{array}$ & 3-HydroxyisovalerylC5-OH $\uparrow$ \\
\hline Methylmalonic acidemia(cobalamin disorders) & Propionyl C3个 \\
\hline Isovalericacidemia & 3-Hydroxyisovaleryl C5-OH $\uparrow$ \\
\hline 3-Methylcrotonyl-CoA carboxylase deficiency & 3-Hydroxyisovaleryl C5-OH $\uparrow$ \\
\hline 3-Hydroxy-3-methyglutaric aciduria & 3-Hydroxyisovaleryl C5-OH $\uparrow$ \\
\hline Holocarboxylasesynthase deficiency & 3-Hydroxyisovaleryl C5-OH $\uparrow$ \\
\hline ß-Ketothiolasedeficiency & Butyryl c4个 \\
\hline Glutaric acidemia type I & Glutaryl C5DC $\uparrow$ \\
\hline \multicolumn{2}{|l|}{ Fatty acid oxidation defect } \\
\hline Carnitine uptake defect/Carnitine transport defect & Free carnitine $\mathrm{C} 0 \downarrow$ \\
\hline $\begin{array}{l}\text { Medium-chain Acyl-CoA dehydrogenase } \\
\text { deficiency }\end{array}$ & Octanoyl C8个 \\
\hline $\begin{array}{l}\text { Very long-chain Acyl-CoA dehydrogenase } \\
\text { deficiency }\end{array}$ & 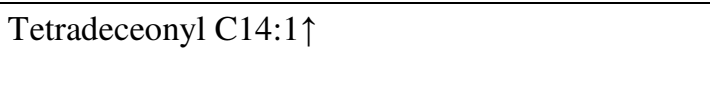 \\
\hline $\begin{array}{l}\text { Long-chain L-3 hydroxyacyl-CoA dehydrogenase } \\
\text { deficiency }\end{array}$ & 3-Hydroxypalmitoyl C16:OH $\uparrow$ \\
\hline Trifunctional protein deficiency & 3-Hydroxypalmitoyl C16:OH $\uparrow$ \\
\hline \multicolumn{2}{|l|}{ Amino acid disorders } \\
\hline Argininosuccinicaciduria & ASA and Citrulline \\
\hline Citrullinemia, type I & Citrulline \\
\hline Homocystinuria & Methionine \\
\hline Tyrosinemia, type I & Tyrosine and Succinyl acetone \\
\hline Classic phenylketonuria & Phenylalanine $[14,15]$ \\
\hline Maple syrup urine disease & Leucine and Isoleucine \\
\hline \multicolumn{2}{|c|}{ Hemoglobin disorders- high performance -liquid chromatography/capillary electrophoresis } \\
\hline Sickle cell anemia(Haemoglobin $\mathrm{S})$ & HB s/s pattern /FS pattern \\
\hline Beta-thalessemia-Hb S & FA pattern \\
\hline Haemoglobin SC disease/variants & FSC pattern \\
\hline
\end{tabular}




\section{Discussion}

The newborn screening and expanded new born screening covering totally covering 64 disorders, which early interventions leads to detect and diagnosis for the prevention of early death / unnoticed disease condition. Newborn dried blood spots are now routinely used for primary screening and research purpose. Since 1960's, pre-analytical techniques are inappropriately considered in the field of newborn screening, while comprises of preparation and processing of DBS for their final analysis has not been standardized. After screening of the samples were stored in freezer for many years for biobanking.

The objective of this study is to identify the early diagnosis and treatment to evaluate clinically with laboratory findings.The results of the DBS have a significantly involved in the mass screening for inborn errors of metabolism in neonates with a desirable strategy. The main advantages to improve accuracy, sensitivity and specificity over existing methods for screening. DBS testing for decades, predominantly focusing on the diagnosis of newborn screening especially in limited resource setting or for high risk strategy screening applications are begin to emerge.

The ideal disorders for screening, at who may appear normal at birth. Theycomprise 31 core disorders currently recommended for state screening programs. DBS testing has been currently in clinical chemistry use [16]. The specific neonatal screening cards $\left(903^{\circledR}\right)$ protein saver cards for this test, with pore size of 0.2 $0.4 \mathrm{um}$, it holds all the enzymes, small and large molecules. For collection of blood, swipe it with $70 \%$ isopropyl alcohol and warm the feet for 10 seconds, the heel prick should be in correct manner, in lateral sides of the heel. In drying of the samples, after collection it should be dried it in a sample rack with the normal room temperature for $2 \mathrm{hrs}$.

When the drying becomes over, the colour of the blood, turned to be in dark brownish colour, which is ready for analysis. Next the sample transportation from one place to another, with the gas-impermeable transparent zipbags with desiccant. Transfer this bag to a freezer with a temperature of $-20 \mathrm{C}$ or if freezer not available in the field store at $-4^{\circ} \mathrm{C}$. The sample elution of DBS is very specific to each analyte quantification. From patients to laboratory testing for screening, the balance residual DBS samples were analysing for research purposes, with obtained consent form and information to the patient.
DBS also suffer from some challenges, with the hematocrit (hct) effect certainly being the most discussed issue. In essence, because of a different viscosity, blood with lower respectively higher het will spread more respectively less than control blood, typically leading to under-respectively overestimation of analyte concentrations when analyzing a DBS subpunch. Furthermore, capillary concentrations can be different from venous concentrations. Other limitations coupled to DBS sampling include the dependence on adequate sampling, risk of contamination, influence of the spotted volume, and spot inhomogeneity.

This imposes a more complicated analytical and clinical validation procedure [17-19]. In addition, due to the small sample size (typically 3-12 $\mu \mathrm{l}$ ) associated with DBS, the amount of analytes and the number of analyses is limited. Therefore, sensitivity requirements may not always be met by available analytical instrumentation.

Serological testing of antibody levels can be used for disease surveillance and diagnostics and generally involves obtaining a sample through a standard blood draw. Performing traditional serological testing requires trained phlebotomists as well as a centrifuge and other supplies to extract the serum from blood specimens soon after collection.

The additional step of repackaging the serum risks labelling errors and specimens transported to a central laboratory for testing must be kept at cool temperatures $\left(4-6^{\circ} \mathrm{C}\right)$.

If serum specimens are stored frozen before shipping, temperatures below $0^{\circ} \mathrm{C}$ must be maintained to avoid antibody-destroying freeze-thaw cycles [20]. The stabilizing effect of DBS - slowing down degradation and/or preventing de novoformation - has also proven to be beneficial in the field of toxicology.

A nice example is the prevention of de novo formation of phosphatidylethanol, a marker indicative of alcohol use, which can be formed ex vivo in blood in the presence of ethanol [21].

In forensic toxicology, DBS may serve as a sample preparation strategy and offer the potential to store evidence of closed cases in a cost-effective way [22,23]. While DBS may be less expensive and easier to collect and transport than serum samples, they can also pose 
Review Article

special challenges to obtain in infants and young children compared to adolescents and young adults. DBS collection requires training and repeated practice to ensure that adequate DBS are collected. In a resource-limited environment or in a scenario where more invasive techniques like venepuncture may be less accepted by the study population, DBS can be the preferred technique to efficiently obtain serum specimens for analyte testing [24]. The features associated with DBS sampling and handling make DBS-based adduct monitoring particularly attractive for large-scale epidemiological studies, an application field that will likely continue to grow in future [25].

\section{Conclusion}

Dried blood spots have been used for infectious disease serology, drug monitoring, environmental investigations and molecular studies. Clinical applications using DBS and the filter paper matrix will continue to expand.

DBS are often the sample of choice in resource-poor areas because of their ease of collection and storage, minimal risk of exposure to blood-borne pathogens and ability to be transported without refrigeration.

As the uses of DBS evolve, so will technologies that make use of new multiplexed methods for DBS applications. If properly performed, it has the ability to provide high-quality results where adequate information cannot be (conveniently) obtained using traditional procedures (e.g. sample collection in remote or resource-limited areas and at patient's homes).

Author Contributions: All authors confirmed they have contributed to the intellectual content of this paper and have met the following requirements: (a) drafting or revising the article for intellectual content; and (b) final approval of the published article.

Acknowledgments: The authors would like to thank Dr. Arasar Seeralar. AT, Dr. Pramila. K and Dr. Sudha Rathna Prabha at Institute of Child health and Hospital for Children, Egmore, Chennai for helpful discussions regarding newborn screening and dried blood spots.

Funding: Nil, Conflict of interest: None initiated, Perission from IRB: Yes

\section{References}

1. Kapoor S. KabraM. Newborn Screening in India: Current Perspectives. Indian Pediatr. 2010;47(3):21924. https://doi.org/10.1007/s13312-010-0043-0
2. Panel discussion on inborn errors of metabolism. The New Indian Express (weekend edition). [cited 2018 Dec 15] Availableat: http://www. newindianexpress.com/ ifestyle/health/2018/dec/15/panel-discussion-on-inbornerrors -of- metabolism-1911764.html

3. De Jesus VR, Zhang XK, Keutzer J, Bodamer OA, Mühl A, Orsini JJ, et al. Development and evaluation of quality control dried blood spot materials in newborn screening for lysosomal storage disorders.ClinChem. 2009;55(1):158-64. doi:10.1373/clinchem.2008.111864.

4. McDade TW, Williams S, Snodgrass JJ. What a drop can do: dried blood spots as a minimally invasive method for integrating biomarkers into populationbased research. Demograph. 2007;44(4):899-925.

5. Mei JV, Alexander JR, Adam BW, Hannon WH. Use of filter paper for the collection and analysis of human whole blood specimens. J Nutr.2001;131(5):1631S-6S. doi:10.1093/jn/131.5.1631S.

6. World Health Organization. (2012). WHO manual for HIV drug resistance testing using dried blood spot specimens, March, 2010 update July, 2012. World Health Organization. https://apps. who. int/iris/ handle/ $10665 / 75829$.

7. Parker SP, Cubitt WD. The use of the dried blood spot sample in epidemiological studies. JClin Pathol. 1999; 52 (9):633-9.doi: 10.1136/jcp.52.9.633

8. Burnett JE. Dried blood spot sampling: Practical considerations and recommendation for use with preclinical studies. Bioanalysis. 2011; 3(10):1099-107. doi: 10.4155/BIO.11.68.

9. Mercader S, Featherstone D, Bellini WJ. Comparison of available methods to elute serum from dried blood spot samples for measles serology. JVirol Methods. 2006; 137(1): 140-9.doi: 10.1016/j.jviromet.2006.06. 018

10. American College of Medical Genetics Newborn Screening Expert Group. Newbornscreening: Toward a uniform screening panel and system - executive summary. Pediatrics2006;117:S296-S307. doi:10.1542/ peds. 2005-2633I.

11.BorremansB.Ammonium improves elution of fixed dried blood spots without affecting immunofluorescence assay quality.Trop Med Int Health. 2014; 19(4):413-6. doi: 10.1111/tmi.12259. Epub 2014 Jan 6. 
Review Article

12. Cassol SA, Read S, Weniger BG, Gomez P, Lapointe N, Ou CY, Babu PG. Dried blood spots collected on filter paper: an international resource for the diagnosis and genetic characterization of human immunodeficiency virus Type-1. Mem. Inst. Oswaldo Cruz.1996; 91(3):351-8.doi: http://dx.doi.org/10. 1590/ S0074- 02761996000300019.

13. Jeffrey DF, Lori MR, Jeremy DR, Paul TS, David $\mathrm{R}$, Ellen KS.State of the science in dried blood spots. Clin Chem. 2018; 64(4):656-79.doi: 10.1373/ clinchem. 2017. 275966.

14. Guthrie R. Blood screening for phenylketonuria. JAMA1961: 178(8):863.doi:10.1001/jama.1961. 03040 470079019

15. Guthrie R, Susi A. A simple phenylalanine method for detecting phenylketonuria in large populations of newborn infants. Pediat. 1963;32(3):338-43.

16. Demirev PA. Dried blood spots: Analysis and applications. Anal Chem. 2013;85(2):779-89.doi: 10. 1021 / ac303205m.

17. Edelbroek PM, van der Heijden J, Stolk LM. Dried blood spot methods in therapeutic drug monitoring: methods, assays, and pitfalls. Ther Drug Monit. 2009;31 (3): 327-36.doi: 10.1097/FTD.0b0 13e31819e91ce.

18. Antunes MV, Charão MF, Linden R. Dried blood spots analysis with mass spectrometry: potentials and pitfalls in therapeutic drug monitoring. Clin Biochem. 2016;49(13-14):1035-46. doi: 10.1016/j.clinbiochem. 2016. 05.004 .
19. De Kesel PM, Sadones N, Capiau S, Lambert WE, Stove CP. Hemato-critical issues in quantitative analysis of dried blood spots: challenges and solutions. Bioanal. 2013;5(16):2023-41. doi: 10.4155/bio.13.156.

20. World Health Organization. Surveillance Guidelines for Measles, Rubella and Congenital Rubella Syndrome in the WHO European Region. Copenhagen, Denmark: WHO Regional Office for Europe; 2012.

21. Kummer N, Lambert WE, Samyn N, Stove CP. Alternative sampling strategies for the assessment of alcohol intake of living persons. Clin Biochem. 2016; 49:1078-91.doi: 10.1016/j.clinbiochem.2016.05.007.

22. Stove CP, Ingels AS, De Kesel PM, Lambert WE. Dried blood spots in toxicology: from the cradle to the grave? Crit Rev Toxicol. 2012;42(3):230-243.doi: 10.3109/10408444.2011.650790.

23. Sadones N, Capiau S, De Kesel PM, Lambert WE, Stove CP. Spot them in the spot: analysis of abused substances using dried blood spots. Bioanalysis. 2014; 6(17):2211-27.doi: 10.4155/bio.14.156.

24. Xu Su, Bradley FC, Xiexiu W.Dried Blood Spots: An evaluation of utility in the field. J Infect Public Health.2018;11(3):373-6.doi:10.1016/j.jiph.2017.09. 014

25. Delahaye L, Janssens B, Stove C. Alternative sampling strategies for the assessment of biomarkers of exposure.Curr OpinToxicol. 2017;4:43-51.doi: https:// doi. org/ 10. 1016/j. cotox.2017.05.003

\section{How to cite this article?}

Subhasree. C. R, Subramaniam S, Shyama S. Biological variations, technical stability and elution tactics in dried blood spots specimens: neonatal screening outcomes. Int J Pediatr Res. 2019;6(07):344-351.doi:10. 17511/ijpr.2019.i07.06 